\title{
DYNAMIC ENHANCED MRI IN DIAGNOSING ADENOMYOSIS BY GRADED APPROACH IN COMPARISON WITH HYSTEROSCOPY BIOPSY CORRELATION
}

Ameen M. D1, Vetriraj $S^{2}$

${ }^{1}$ Associate Professor, Department of Radiodiagnosis.

${ }^{2}$ Associate Professor, Department of Radiodiagnosis.

\begin{abstract}
BACKGROUND
ABSTRACT

Adenomyosis is not an uncommon condition on routine ultrasonography. Specificity of ultrasound in adenomyosis is non-specific, probably due to inadequate bladder preparation and non-specific history background. In common, late stage adenomyosis is considerably diagnosed on ultrasound. Therefore, emphasis on importance of MRI in adenomyosis by standardised grading and early diagnostic algorithm is challenging in future prospects.

The objective of this study is to diagnose different grades of adenomyosis from early to late changes and to compare MRI grading with grading by hysteroscopy biopsy.
\end{abstract}

\section{MATERIALS AND METHODS}

We have decided the sample size of 49 , calculated based on previous one year record in our hospital. Ultrasound was done (n=49) and positive clinical background patients were included for MRI and further hysteroscopy biopsy. Hysteroscopy biopsy grading was done within 2 weeks follow-up and considered as Gold Standard. Based on grades of myometrial fibrous tissue involvement, results were compared. MRI reports were done by one experienced radiologist.

\section{RESULTS}

All positive patients were studied and graded by MRI protocol. MRI graded approach shows better sensitivity for grades (Grades II and IV) as compared with (Grade I and III). The biopsy vs. MRI result showed that normal Grade I, Grade II, Grade III and Grade IV were $13.8 \%$ vs. $17.2 \%$; $41.4 \%$ vs. 44.5 ; 31.0 vs. 31.0 ; 3.4 vs. 3.4 and 3.4 vs. 3.4 respectively.

\section{CONCLUSION}

MRI graded approach algorithm as compared to routine MRI for adenomyosis proved to be a valid tool in future prospective; however, false positive results exist in higher grades.

\section{KEYWORDS}

Adenomyosis; MRI Grading; Hysteroscopy Grading; Ultrasound.

HOW TO CITE THIS ARTICLE: Ameen MD, Vetriraj S. Dynamic enhanced MRI in diagnosing adenomyosis by graded approach in comparison with hysteroscopy biopsy correlation. J. Evolution Med. Dent. Sci. 2018;7(01):20-23, DOI: 10.14260/jemds/2018/6

\section{BACKGROUND}

Uterus anatomy on the basis can be divided into two clearly distinct parts: the endometrium and myometrium. In 1983, Hricak et al(1) delineated the normal zonal anatomy on MRI that was confirmed by other authors. ${ }^{(2-5)}$ The term "junctional zone" was introduced in this manner to describe this interface observed on MRI: a distinct low signal on T2weighted sequences separating the endometrium in high signal intensity from the outer myometrium in intermediate signal. Pelvic MRI was ahead of anatomical-histological methods, which on light microscopy could not identify this uterine zonal anatomy.(6) Subsequently, it was clearly shown that this zone corresponds to the innermost layer of the myometrium and not to the basal layer of the endometrium.(7) Since the publication by Hricak et al,(1) numerous groups have studied zonal anatomy of the uterus on T2-weighted imaging, most notably the low signal intensity of the junctional zone.(8-10)

'Financial or Other Competing Interest': None.

Submission 05-12-2017, Peer Review 20-12-2017

Acceptance 23-12-2017, Published 01-01-2018.

Corresponding Author:

Dr. Ameen M. D

Flat $1 C$, No. 9,

Swaraj Manor Leithe Castle Centre Street,

Santhome, Chennai-600028.

E-mail: drameenmdrd@rocketmail.com

DOI: $10.14260 /$ jemds $/ 2018 / 6$
These works have precisely correlated the MRI data with those obtained on detailed anatomical-histological examination. The junctional zone has been shown to contain myocytes with morphologic characteristics differing from those of the typical myocytes of the outer myometrium. Junctional zone myocytes present a greater relative nuclear area, a looser extracellular matrix and lower water content.(8) These elements serve to decrease the signal of this zone on T2-weighted imaging, although the different morphology of myocytes is not the sole cause. The architectural organisation of the inner myometrium is unique with a concentric arrangement of smooth-muscle fibres in contrast to the longitudinal orientation of the smooth-muscle fibres of the outer myometrium.(10) To better understand the histologic factors that explain the signal characteristics of the junctional zone, it is pertinent to objectively evaluate healthy control subjects.

The simplest and most studied measure is the thickness of junctional zone, which is defined as the subendometrial low signal band.(1-4,11) The thickness of this layer is crucial, because above certain threshold it is a strong criterion for the diagnosis of adenomyosis. To be reproducible, the junctional zone measurement should be made on a mid-sagittal image through the long axis of the uterus. There is physiological variation in junctional zone due to pregnancy and due to hormonal cycle changes. 
Adenomyosis is not an uncommon condition on routine ultrasonography. On most of routine ultrasound, early adenomyosis without proper clinical history with positive finding is missed. Specificity of ultrasound in adenomyosis is non-specific, probably due to inadequate bladder preparation and nonspecific history background. Only late stages adenomyosis was considerably diagnosed on ultrasound in common. Therefore, emphasis on importance of MRI in adenomyosis by standardised grading and early diagnostic algorithm is challenging in future prospects. From literature background, direct signs and indirect signs of adenomyosis was described on MRI. Direct signs are microcysts/ adenomyoma detection. Indirect signs are thickness of junctional zone. However, this information lacks specificity in routine scenario as adenomyoma classification is nonspecific.

To the best of our knowledge various grading approach exists without standardised approach and grouping into 4 Grades in correlation with hysteroscopy biopsy grades our study illustrated specific nature.

\section{Objectives}

1. To diagnose different grades of adenomyosis from early to late changes, and

2. To compare MRI grading of adenomyosis with grading by Hysteroscopy biopsy.

\section{MATERIALS AND METHODS}

Number of diagnosed adenomyosis patients attended in Radiology department in our hospital during last year was 44 . We expected it to increase by $10 \%$ in the current year. So based on this, sample size was calculated as 49 . Patients with positive clinical background were enrolled in the study. Ultrasound was subjected at baseline for all 49 patients. Hysteroscopic biopsy and MRI were done for all ultrasonography positive patients. Further, all USG negative patients were subjected to MRI selective screening protocol. Again, if negative result, then were further subjected to hysteroscopy biopsy. Various grades of adenomyosis results were obtained following MRI and biopsy. Hysteroscopy biopsy grading was done within 2 weeks and considered as Gold Standard based on myometrial fibrous tissue involvement and graded. The MRI reports were done by experienced radiologist. Informed consent was obtained from all patients and information so collected were kept confidential and anonymous.

\section{Statistical Analysis}

The collected data were analysed with SPSS software 23.0 Version.

\section{Image Protocol}

MRI 1.5 Tesla Siemens unit with dedicated 16-channel body coil deployed. Routine T2 and T1 W sequence and diffusion/ $\mathrm{ADC}$ and fat sat and gradient sequence also included in protocol in three plane sections were covered without contrast study.

\section{RESULTS}

Biopsy grading was also done in our study and grouped into 4 categories as to compare with MRI grading. Grade 0- normal myometrial fibrous tissue. Grade I- minimal increase in myometrial fibrous tissue. Grade II- moderate increase in myometrial fibrous tissue. Grade III- moderate increase in myometrial fibrous tissue with large cystic vacuolation. Grade IV- diffuse massive increase in myometrial fibrous tissue with thickened stroma showing biopsy versus MRI. The biopsy vs. MRI result showed that normal Grade I, Grade II, Grade III and Grade IV were $13.8 \%$ vs. $17.2 \%$; $41.4 \%$ vs. $44.5 ; 31.0$ vs. 31.0 ; 3.4 vs. 3.4 and 3.4 vs. 3.4 respectively (Table 1). Grade III category three patients were in biopsy pickup, whereas on MRI one patient was categorised.

Results were mismatched in MRI grades as compared to Gold Standard hysteroscopy due to the fact that size criteria kept was above $3 \mathrm{~mm}$ for adenomyosis or adenomyoma cyst. Biopsy taken was only fibrous tissue criteria, specifically not adenomyoma cyst probably. In Grade IV likewise, MRI correlated with biopsy due to the fact that of one patient the criteria were bulky myometrium and junctional zone interface and no adenomyoma cyst was included. The biopsy vs. MRI result showed that normal Grade I, Grade II, Grade III and Grade IV were $13.8 \%$ vs. $17.2 \% ; 41.4 \%$ vs. $44.5 ; 31.0$ vs. $31.0 ; 3.4$ vs. 3.4 and 3.4 vs. 3.4 respectively. The $(\mathrm{N}=20)$ negative USG patients are subjected to MRI selective screening protocol of sagittal T2W images showing no positive results Grade 0 (normal); hence, those patients are further subjected to hysteroscopy biopsy. MRI graded approach shows better sensitivity for grades (Grades II and IV) as compared with (Grade I and III). In our study, most of the MRI grades fall into Grade II, moderate sized microcysts > $3 \mathrm{~mm}$. Due to Superior T2W image, temporal resolution and SNR ratio is good. Biopsy also correlated with most of the pathology by Grades II criteria; however, pit fall in biopsy is adenomyoma cyst size criteria not specific. That is where correlation mismatched results obtained in Grade III.

\section{Image Analysis and Interpretation}

Figure 3, four grades of analysis as the graph showing MRI graded approach with Biopsy correlation was normal, Grade I-IV study. By Biopsy grading our study was classified as follows- normal- no evidence of junctional zone anatomy. Grade I- minimal microcysts criteria. Grade II- moderate microcysts criteria $>3 \mathrm{~mm}$. Grade III- Large microcysts in junctional zone $>3 \mathrm{~mm}$ with diffuse increase in junctional zone and myometrial interface $<10 \mathrm{~mm}$. Grade IV- Bulky myometrium junctional zone interface $>10 \mathrm{~mm}$.

\begin{tabular}{|c|c|c|c|}
\hline Grades & $\begin{array}{c}\text { Biopsy } \\
\text { N (\%) }\end{array}$ & $\begin{array}{c}\text { MRI Grades } \\
\text { N (\%) }\end{array}$ & $\begin{array}{c}\text { Total N } \\
\text { (\%) }\end{array}$ \\
\hline Normal & $4(13.8)$ & $5(17.2)$ & $15.6 \%$ \\
\hline I & $12(41.4)$ & $13(44.8)$ & $42.7 \%$ \\
\hline II & $9(31.0)$ & $9(31.0)$ & $31.0 \%$ \\
\hline III & $1(10.3)$ & $1(3.4)$ & $6.9 \%$ \\
\hline IV & $1(3.4)$ & $1(3.4)$ & $3.4 \%$ \\
\hline \multicolumn{4}{|c|}{ Table 1. Grading of Adenomyosis by Hysteroscopic } \\
Biopsy and MRI \\
\hline
\end{tabular}



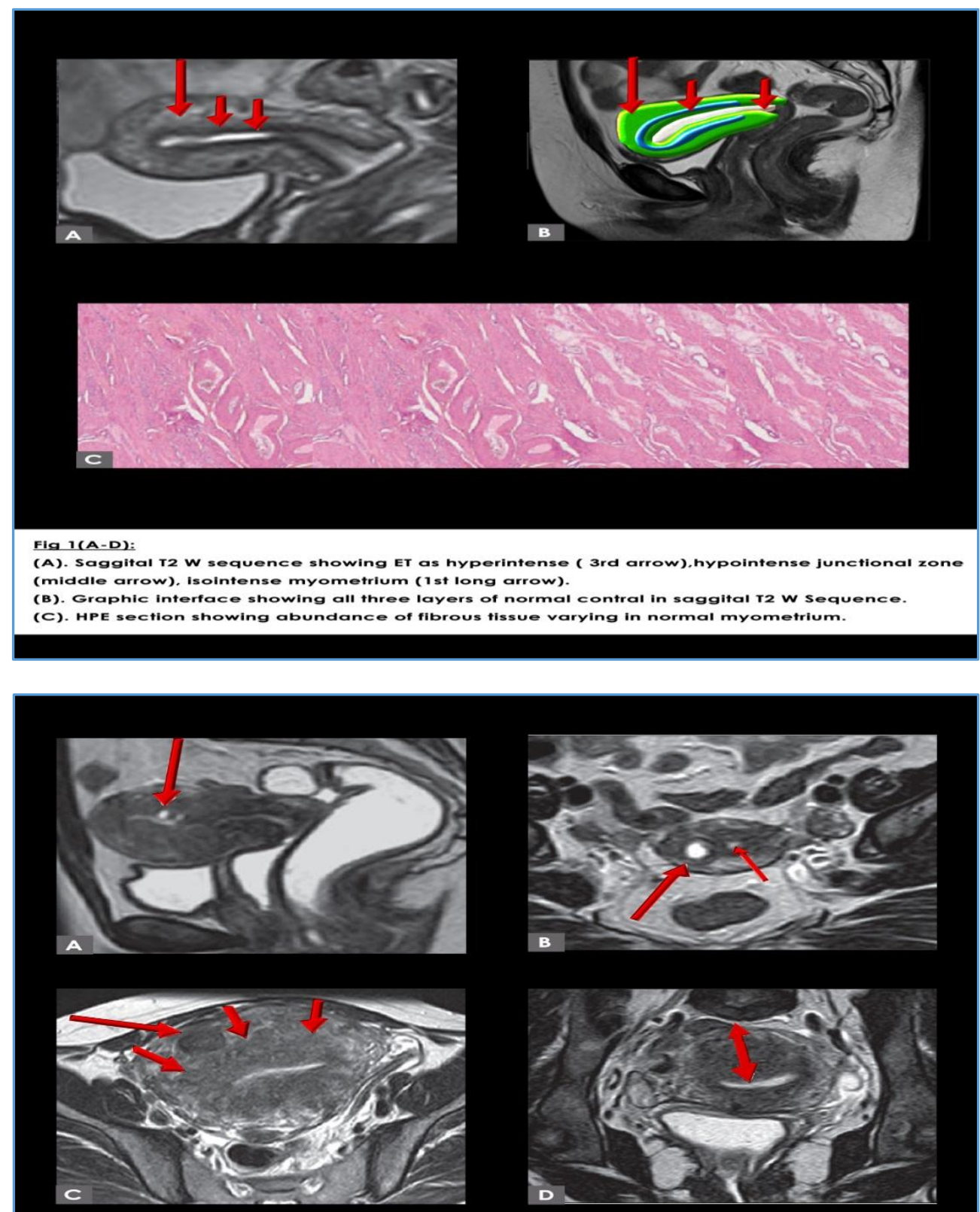

\section{Fig 2(A-D):}

(A). Saggital $\mathrm{T} 2 \mathrm{~W}$ sequence showing grade I adenomyosis with microcyst formation.

(B). Axial $12 \mathrm{~W}$ sequence showing grade ll adenomyosis with microcysts $>3 \mathrm{~mm}$.

(C). Axial $12 \mathrm{~W}$ Sequence showing grade III adenomyosis with microcysts $>4 \mathrm{~mm}$ and diffuse

increased myometrial interface.

(D) Coronal Axial T2 W sequence showing bulky junctional zone \& myometrial interface.

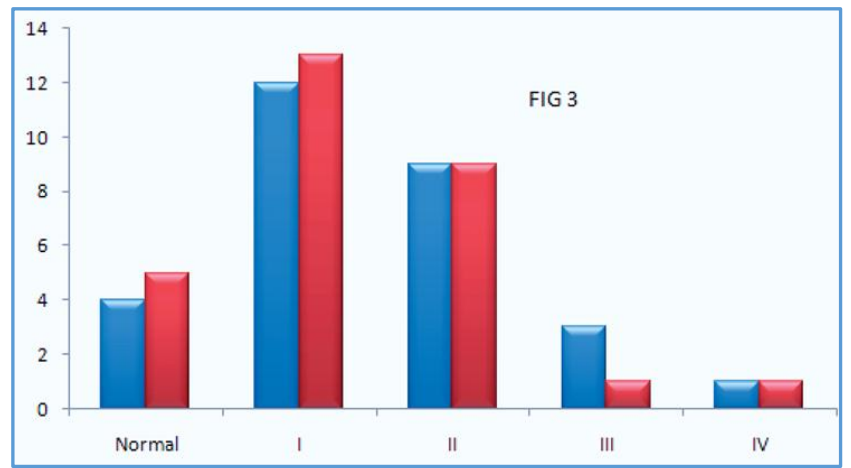

Figure 3. Four Grades of Analysis showing MRI Graded Approach with Biopsy Approach

\section{DISCUSSION}

Combined with baseline ultrasonography can yield better sensitivity of $78.94 \%$ and specificity of $87.38 \%$. These results were due to correlation with hysteroscopy biopsy as Gold Standard. Moreover, biopsy could not grade microcysts' size criteria specifically as MRI does, especially in Grade III scenario. In future prospects, MRI can be the Gold Standard than any other invasive or non-invasive study in grading adenomyosis by size criteria. As Figure 1 is illustrating Grade 0 - (Normal grade), endometrium hyperintense in nature, junctional zone hypointense in nature and myometrium isointense in nature in standard $\mathrm{T} 2 \mathrm{~W}$ sequence. As compared to figure Grades 1 to 4 showing Grade I- minimal microcysts criteria, Grade II- moderate microcysts criteria $>3 \mathrm{~mm}$. Grade 
III- Large microcysts in junctional zone was $>3 \mathrm{~mm}$ with diffuse increase in junctional zone and myometrial interface was < $10 \mathrm{~mm}$. Grade IV- Bulky junctional zone and myometrium interface was $>10 \mathrm{~mm}$. This customised MRI graded approach included in our study, to the best of our knowledge we standardised it. In future prospects by large cohort study, this MRI graded approach efficacy should be proven with any other modality. In our case, microcysts in junctional zone with size criteria was given in varying range from 2 to $6 \mathrm{~mm}$. Since the term adenomyoma is a histological term, in our study we did not coin the cysts in such manner, rather the size of the cyst was only given at each grade. Hysterosalpingography no longer serves a major role in the diagnosis of adenomyosis. This more invasive imaging procedure has been largely supplanted by abdominal ultrasound followed by endovaginal ultrasound. The advantages of ultrasound are ease of use on a daily basis and diagnostic performance when exploration is of good quality. However, MRI has evolved as the current imaging technique of choice in confirming suspected cases of adenomyosis. To this end, several signs of adenomyosis- both direct and indirect- have been described.(3,4,5) In our study, Figure 2 is showing different grades from our study collection of adenomyoma. An adenomyoma is composed of a focal consolidation of adenomyotic glands located between the two entities. Although, both adenomyoma and leiomyoma are of low signal intensity on T2-weighted imaging, adenomyoma often will have high-signal intensity foci on T2. Moreover, in contrast to other myomas, adenomyoma does not have large vessels at its periphery. Adenomyoma is rarer than both focal and diffuse adenomyosis, although its true prevalence is difficult to establish. Another study isolated only one case among 28 patients with adenomyosis, whereas Bazot et al reported seven cases among 40 surgically proven cases. Cases of cystic adenomyosis corresponding to an excessive haemorrhage of ectopic endometrium within the myometrium have also been described. Such lesions present as a cavity with a long axis of greater than $1 \mathrm{~cm}$ that contains haemorrhagic or liquid contents surrounded by a fibrous tissue with hypointense signal on T2-weighted imaging.

\section{CONCLUSION}

MRI graded approach algorithm as compared to routine MRI for adenomyosis proved to be a valid tool in future prospective; however, false positive results exist in higher grades. Combined with baseline ultrasonography can yield better sensitivity and specificity.

\section{ACKNOWLEDGEMENT}

We thank Venkatesan Zigma, PhD, for helping with Statistical Analysis.

\section{REFERENCES}

[1] Lee JK, Gersell DJ, Balfe DM, et al. The uterus: in vitro MR-anatomic correlation of normal and abnormal specimens. Radiology 1985;157(1):175-9.

[2] Hricak H, Alpers C, Crooks LE, et al. Magnetic resonance imaging of the female pelvis: initial experience. AJR 1983;141(6):1119-28.

[3] Holliday J, Saxon R, Lufkin RB, et al. Anatomic correlations of magnetic resonance images with cadaver cryosections. Radiographics 1985;5(6):887921.

[4] Arrivé L, Guinet C, Buy JN, et al. Magnetic resonance imaging of the pelvic area: preliminary results [in French]. J Radiol 1985;66(12):771-7.

[5] Bryan PJ, Butler HE, Li Puma JP. Magnetic resonance imaging of the pelvis. Radiol Clin North Am 1984;22(4):897-915.

[6] Fusi L, Cloke B, Brosens JJ. The uterine junctional zone. Best Pract Res Clin Obstet Gynaecol 2006;20(4):47991.

[7] Scoutt LM, Flynn SD, Luthringer DJ, et al. Junctional zone of the uterus: correlation of MR imaging and histologic examination of hysterectomy specimens. Radiology 1991;179(2):403-7.

[8] McCarthy S, Scott G, Majumdar S, et al. Uterine junctional zone: MR study of water content and relaxation properties. Radiology 1989;171(1):241-3.

[9] Mitchell DG, Schonholz L, Hilpert PL, et al. Zones of the uterus: discrepancy between US and MR images. Radiology 1990;174(3 Pt 1):827-31.

[10] Brown HK, Stoll BS, Nicosia SV, et al. Uterine junctional zone: correlation between histologic findings and MR imaging. Radiology 1991;179(2):409-13.

[11] Demas BE, Hricak H, Jaffe RB. Uterine MR imaging: effects of hormonal stimulation. Radiology 1986;159(1):123-6. 